\title{
Alterstice
}

Revue internationale de la recherche interculturelle

International Journal of Intercultural Research

Revista International de la Investigacion Intercultural

\section{Le patrimoine au défi de l'interculturalité : enjeux et nouvelles pratiques}

\section{Claire Autant-Dorier}

Volume 5, numéro 2, 2015

Patrimoine et interculturalité

URI : https://id.erudit.org/iderudit/1036687ar

DOI : https://doi.org/10.7202/1036687ar

Aller au sommaire du numéro

Éditeur(s)

Alterstice

ISSN

1923-919X (numérique)

Découvrir la revue

Citer ce document

Autant-Dorier, C. (2015). Le patrimoine au défi de l'interculturalité : enjeux et nouvelles pratiques. Alterstice, 5(2), 7-19. https://doi.org/10.7202/1036687ar

\section{Résumé de l'article}

Le patrimoine semble être devenu un outil de promotion, voire de " gestion " de la diversité, notamment au niveau des instances politiques internationales. Il serait un opérateur de construction du « commun ». La première partie de l'article pointe le fait que la seule reconnaissance de la diversité ne saurait toutefois suffire à faire du commun, car elle maintient une conception identitaire, voire réifiée, du patrimoine. L'hypothèse est faite que, pour devenir " commun ", le patrimoine devrait être désapproprié, y compris dans sa dimension publique. L'auteur examine alors comment les articles de ce numéro déclinent diverses conceptions et modalités de faire " patrimoine commun ". Il apparaît que les sources de production et de légitimation du patrimoine se diversifient, leurs manifestations et formes changent, en particulier depuis la reconnaissance du patrimoine culturel immatériel, et les acteurs eux-mêmes s'hybrident. L'analyse porte, dans une deuxième partie, sur la façon dont les voix profanes et minorées contribuent à une co-activité de définition de la légitimité et des usages de ce qui, dès lors, fait patrimoine. Il y aurait là basculement de régime patrimonial, dans lequel plus que d'authentification, il s'agirait d'un travail politique de reconsidération. Celui-ci passe par des expériences, voir des expérimentations, dans lesquelles le chercheur est également un acteur impliqué. La dernière partie identifie, au travers des contributions présentées, quelques caractéristiques du patrimoine mis à l'épreuve de l'interculturalité. Ce qui fait la force du patrimoine, en régime interculturel, ne dépendrait pas de procédures d'authentification, de classement, de protection, mais des performances qui l'actualisent et de sa capacité prospective. Des espaces d'invention de la culture sont ainsi activés.
Ce document est protégé par la loi sur le droit d'auteur. L’utilisation des services d’Érudit (y compris la reproduction) est assujettie à sa politique d'utilisation que vous pouvez consulter en ligne.

https://apropos.erudit.org/fr/usagers/politique-dutilisation/ 


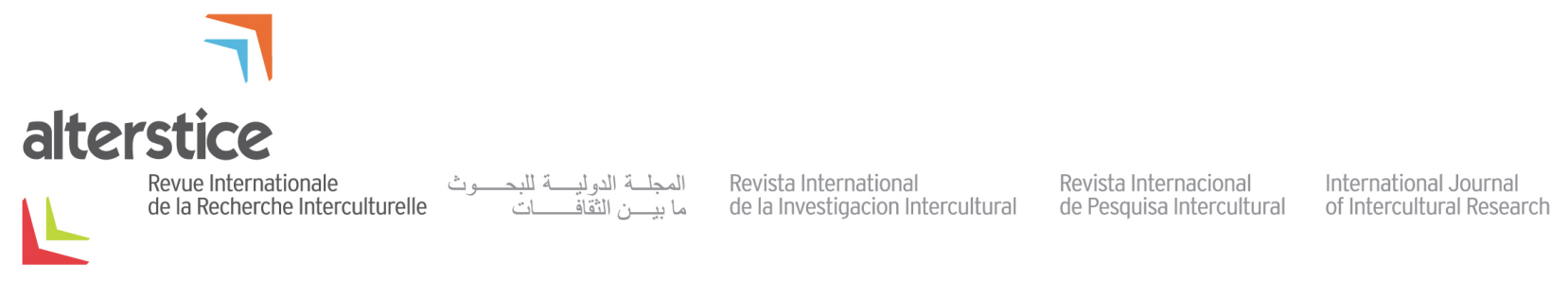

INTRODUCTION THÉMATIQUE

\title{
Le patrimoine au défi de l'interculturalité : enjeux et nouvelles pratiques
}

\author{
Claire Autant-Dorier ${ }^{1}$
}

\section{Résumé}

Le patrimoine semble être devenu un outil de promotion, voire de " gestion » de la diversité, notamment au niveau des instances politiques internationales. Il serait un opérateur de construction du « commun ». La première partie de l'article pointe le fait que la seule reconnaissance de la diversité ne saurait toutefois suffire à faire du commun, car elle maintient une conception identitaire, voire réifiée, du patrimoine. L'hypothèse est faite que, pour devenir " commun », le patrimoine devrait être désapproprié, y compris dans sa dimension publique. L'auteur examine alors comment les articles de ce numéro déclinent diverses conceptions et modalités de faire "patrimoine commun ». Il apparaît que les sources de production et de légitimation du patrimoine se diversifient, leurs manifestations et formes changent, en particulier depuis la reconnaissance du patrimoine culturel immatériel, et les acteurs eux-mêmes s'hybrident. L'analyse porte, dans une deuxième partie, sur la façon dont les voix profanes et minorées contribuent à une co-activité de définition de la légitimité et des usages de ce qui, dès lors, fait patrimoine. Il y aurait là basculement de régime patrimonial, dans lequel plus que d'authentification, il s'agirait d'un travail politique de reconsidération. Celui-ci passe par des expériences, voir des expérimentations, dans lesquelles le chercheur est également un acteur impliqué. La dernière partie identifie, au travers des contributions présentées, quelques caractéristiques du patrimoine mis à l'épreuve de l'interculturalité. Ce qui fait la force du patrimoine, en régime interculturel, ne dépendrait pas de procédures d'authentification, de classement, de protection, mais des performances qui l'actualisent et de sa capacité prospective. Des espaces d'invention de la culture sont ainsi activés.

\section{Rattachement de l'auteure \\ ${ }^{1}$ Université Jean Monnet, Saint-Étienne, France.}

\section{Correspondance}

claire.autant@wanadoo.fr

\section{Mots clés}

patrimoine commun, interculturalité, expérience, activation, invention, patrimoine impropre.

\section{Pour citer cet article}

Autant-Dorier, C. (2015). Le patrimoine au défi de l'interculturalité : enjeux et nouvelles pratiques. Alterstice, 5(2), 7-19. 


\section{Préambule}

Ce numéro de la revue Alterstice est mené en collaboration avec le Groupement d'intérêt scientifique Institutions patrimoniales et pratiques interculturelles (GIS IPAPIC, www.ipapic.eu) qui associe des musées, des archives et des bibliothèques ainsi que des laboratoires et des associations afin de partager les expériences et de conduire les réflexions sur les dynamiques interculturelles dans les processus patrimoniaux.

L'interculturalité n'est pas affirmée par les membres du GIS comme un principe, mais posée comme un objet de questionnement, matière de dissensus plutôt que de consensus. Elle est cet espace tiers, cet " entre ", où le commun peut se construire à partir des diversités et des différends. Ainsi, ce ne sont pas les cultures qui dialoguent entre elles, mais bien des individus entre eux. Les cultures sont ainsi pensées comme n'étant jamais des totalités homogènes et monolithiques, et c'est en ce sens que la notion de " pratiques interculturelles » est privilégiée.

Cette perspective rejoint pleinement celle de la revue Alterstice, qui invite à être attentif aux modalités de l'inter et aux jeux de frontières entendus comme espace de porosités. Les pratiques de traductions, d'interprétations, d’hybridations sont ainsi au centre des questionnements poursuivis et des travaux présentés.

\section{Introduction}

Portées par les mobilités multiples (du travail, de la formation, du tourisme, des migrations économiques et politiques), par les réseaux virtuels et la numérisation, les pratiques interculturelles redessinent les territoires et recomposent les patrimoines entre histoire, mémoire et temps présent, entre nature et culture. Un double défi émerge ainsi :

- le défi de l'interculturalité, posé par la reconnaissance de la diversité des formes d'expressions culturelles, la multiplication et la diversification des échanges dans le monde contemporain, ainsi que par la complexification des sociétés et les changements dans les pratiques culturelles,

- le défi du patrimoine et des processus de patrimonialisation, posé par l'extension de la notion de patrimoine et par les demandes de reconnaissance sociale et politique qui s'y trouvent impliquées, notamment en matière de représentations, d'enjeux mémoriels ou de politiques de la mémoire. Ces revendications, et les initiatives qui les accompagnent, posent la question à la fois de la diversité des patrimoines et de la prise en compte des différences, au sens plus hiérarchique que donne Bhabha Homi (2007) à cette notion.

Comme nous allons le voir, le patrimoine semble être devenu aujourd'hui un outil de promotion, voire de " gestion » de la diversité, notamment au niveau des instances politiques internationales. II serait un opérateur de construction du «commun ». Dans le même mouvement, des voix minoritaires, périphériques ou discordantes font émerger des opportunités nouvelles ou y répondent. Les sources de production et de légitimation du patrimoine tendent à se diversifier. Et leurs manifestations et formes changent, en particulier depuis la reconnaissance du patrimoine culturel immatériel. Enfin, les acteurs eux-mêmes s'hybrident. Ces expériences ont imposé d'explorer de nouvelles conceptions du "faire patrimoine ", aussi bien sur le plan des pratiques que sur le plan conceptuel. Les travaux qui se développent autour de ces questions, et dont les articles proposés ici témoignent, s'intéressent alors davantage au patrimoine comme une " activité » que comme un « objet ».

Ce numéro propose d'examiner en quoi l'interculturalité prend place dans les activités patrimoniales. Comment les personnes et les collectifs concernés ainsi que leurs interlocuteurs construisent-ils des pratiques interculturelles dans des activités ou des processus patrimoniaux? Réciproquement, comment la dimension patrimoniale est-elle activée dans les pratiques interculturelles? Qu'est-ce que cela suppose de reconsidérer? Finalement, peut-on " patrimonialiser l'interculturalité » ou parler de « patrimoine interculturel »?

\section{Le patrimoine, instrument de la construction du commun}

Depuis les années 2000, avec la déclaration de l'UNESCO sur la diversité culturelle comme patrimoine commun de I'humanité (en 2001), la convention de Faro en Europe (en 2005) qui affirme la valeur culturelle du patrimoine pour 
la société, puis l'année européenne du dialogue culturel (en 2008), les instances internationales qui se donnent pour mission de favoriser la paix et de développer un « vivre ensemble » de qualité paraissent s'accorder sur le fait que la culture dans sa diversité peut faire patrimoine commun. Elles y voient la possibilité de faire lien et sens audelà des situations de crises économique, écologique et politique que les sociétés post-modernes sont en train de traverser.

La notion de diversité est considérée dans le discours institutionnel et politique progressiste comme un atout, découlant à la fois de la pluralité interne de nos sociétés modernes et de la reconnaissance du caractère pluriel du Sujet social. Cette reconnaissance et cette valorisation de la diversité sont en même temps au fondement d'un discours politique visant à renforcer la cohésion sociale par l'idéologie du vivre ensemble et de l'égalité des chances, qui sont censés faire reculer les inégalités et les discriminations. Dans cette optique, la " gestion de la diversité » renvoie essentiellement au domaine du politique, autour des concepts d'intégration et de respect du droit des groupes minoritaires (femmes, homosexuels, handicapés, étrangers) dans leur rapport au groupe dominant représenté par les instances étatiques. (Goguikian Ratcliff et Rossi, 2014, p. 8)

Tout se passe comme si les cultures une fois faites patrimoine garantissaient le dialogue entre individus et groupes humains. Il s'opérerait là une reconnaissance de chacun propice à dépasser les injustices comme les jeux d'intérêts et de pouvoir. La montée en reconnaissance en tant que patrimoine, et qui plus est en tant que patrimoine mondial, conférerait ainsi à la culture de chacun légitimité et place. La création de la notion de patrimoine culturel immatériel (2003) a eu précisément pour vocation d'élargir les conceptions du patrimoine et de faire place à des " communautés » qui n'avaient pas pu faire valoir leurs contributions culturelles auparavant (Bortolotto, 2011). Mais, même " élevées " au rang de patrimoine, les cultures toutes seules ne font rien. C'est bien l'ensemble des discussions, négociations et traductions qui peuvent permettre de reconnaître la diversité des cultures comme patrimoine commun, et ce, quel que soit le niveau auquel on se situe : à l'échelle mondiale comme à l'échelle des interactions ordinaires.

Les recherches sur l'interculturalité, comme les expériences de formation en ce domaine, ont déjà montré l'importance de ne pas s'en tenir à une simple reconnaissance des coutumes et traditions de l'Autre comme autant de traits culturels spécifiques. C'est dans un travail de décentrement, d'interrogation réciproque sur nos cadres de références respectifs, et surtout dans un travail commun d'élaboration des significations, que s'opère la rencontre interculturelle (Cohen-Emerique et Rothberg, 2015). Reste à examiner plus avant ce que l'on entend par " patrimoine commun » et la façon dont la diversité y trouve place.

Le patrimoine a d'abord été défini au travers de ses qualités intrinsèques (inventaire, classification), mais les travaux sur les processus de patrimonialisation ont insisté sur l'attention à accorder à ce qui se passe dans les différentes opérations qui fabriquent le patrimoine: découverte, connaissance, authentification, déclaration, transmission (Davallon, 2006) et sur les effets de " rupture " que l'institution du patrimoine produit (Rautenberg, 2003). Par ailleurs, on assiste depuis plus de 30 ans à la multiplication des petits patrimoines (Micoud, 1999). Les recherches conduites sur ces phénomènes ont permis d'identifier la façon dont les groupes manifestent par là leur identité, œuvrent à la faire reconnaître et font usage des ressources que cela leur procure, notamment en matière de développement touristique. Si l'émergence de ces dynamiques patrimoniales multiples permet une diversification de ce qui fait patrimoine, elles sont parfois le support de définitions identitaires réductrices qui s'éloignent de la perspective d'une contribution au « commun ».

Reconnaître la diversité des patrimoines ne conduit ainsi pas nécessairement à reconnaître la diversité dans le patrimoine, soit la dimension interculturelle inscrite dans les "faits " culturels et au sein des processus de patrimonialisation eux-mêmes. Par ailleurs, cette reconnaissance de la diversité peut également demeurer partielle, en ne retenant que ce qui contribue à nourrir un discours unificateur. Ainsi, en France, la montée en puissance des travaux sur les mémoires de l'immigration a permis de faire valoir une multiplicité de récits, mais demeure fortement marquée par la perspective de l'intégration : du côté institutionnel, il s'agit de prendre en compte dans le récit national la contribution des immigrés à la Nation plus que de rendre compte des expériences migratoires et des complexités territoriales et identitaires dans leur ensemble. La Cité nationale, devenue musée de I'histoire de l'immigration, illustre bien le cadre étroit dans lequel cette prise en compte s'est opérée. Elle a été inaugurée plusieurs années après son ouverture effective, et sans que la colonisation y soit réellement abordée au- 
delà de l'histoire du lieu (le Palais de la Porte Dorée). Du côté des initiatives locales, les projets mémoriels qui se donnent souvent un objectif de cohésion sociale comportent une dimension culturaliste :

On renferme encore les individus sur un groupe, cette fois résidentiel, alors que les frontières spatiales de ces quartiers viennent approximativement se substituer aux frontières ethniques ou communautaires. On renforce la place du quartier dans la construction des identités sociales au détriment de ce que pourraient être des mémoires du travail, des usages de la ville, des réseaux, etc. (Rautenberg, 2006, p. 13).

De ce point de vue, il convient de relever un paradoxe inhérent aux définitions et critères qui s'appliquent au patrimoine : il réfère à des " communautés " spécifiques, considérées comme en étant détentrices ou porteuses. Ainsi, les premiers critères retenus pour le patrimoine culturel immatériel $(\mathrm{PCl})$ sont: premièrement, que les éléments patrimoniaux soient " reconnus comme faisant partie de leur patrimoine culturel par des communautés, des groupes et, le cas échéant, des individus " et, deuxièmement, qu'ils soient "transmis de génération en génération » (Unesco, 2003). Les phénomènes d'emprunts, d'acculturation, de métissage, de réinterprétations ne sont pas évoqués, alors même que l'on sait le rôle essentiel que l'on fait jouer au patrimoine dans l'invention des traditions (Hobsbawm et Ranger, 1983) et l'importance actuelle des connexions plus horizontales. Ce qui fait valeur universelle pour l'humanité, c'est la reconnaissance de son existence singulière, de sa contribution à la diversité, mais le caractère interculturel d'un patrimoine en soi ne semble pas être envisagé ${ }^{1}$. Seule la convention de Faro, qui insiste sur la contribution au processus de patrimonialisation, ne prédéfinit pas l'objet patrimonial en tant que tel. Brianso (dans ce numéro) note que la convention de Faro ne ferme pas la notion de patrimoine mais l'ouvre, en proposant une conception dynamique et en insistant sur la volonté de participation. Au point que la notion de " patrimoine commun de l'Europe " n'est pas associée dans les textes à ce qui caractériserait son " identité réelle». Cela introduit la possibilité de considérer, et même d'encourager, les créations patrimoniales qui ne soient pas exclusives à un groupe ou à une seule communauté2.

Cette tension entre construction d'un commun qui dépasse les logiques d'appartenance exclusive et la notion de propriété ou d'appropriation du patrimoine est présente dès l'origine de la notion. Comme le note Davallon :

C'est simplement dans les modifications - les juristes connaissent ça - qui sont intervenues dans la conception du patrimoine que la notion de bien commun est restée comme, d'ailleurs, l'ont analysé Babelon et Chastel (1994), dans leur article célèbre sur la définition de patrimoine. Ils ont montré que c'était l'Église qui, à travers le patrimoine de Saint-Pierre, avait conservé l'idée d'un bien commun, qui n'était pas appropriable par les individus. On arrive à ce paradoxe, que ce que nous appelons patrimoine culturel, en fait, n'est pas du patrimoine au sens commun. C'est-à-dire que ce sont des choses qui ne peuvent pas être appropriées par les individus. Cela ne peut pas faire partie des biens d'une personne, qu'elle peut léguer ou qu'elle va pouvoir vendre et donc disposer de la manière dont elle l'entend. C'est une première chose, qui pose la question de savoir ce que c'est, au fond, que cette catégorie extrêmement curieuse de patrimoine culturel, qu'il soit matériel ou immatériel d'ailleurs. (2013, p. 2)

Cette analyse, faite selon un angle juridique et sémiotique, demande à être prolongée et dépassée pour envisager la question du patrimoine commun en regard de l'interculturalité. Nous faisons l'hypothèse que, pour devenir "commun », le patrimoine devrait être désapproprié, y compris dans sa dimension publique. De fait, les conceptions réifiées et identificatrices du patrimoine ne permettent pas de l'envisager comme un espace de véritable dialogue interculturel. Rendre le patrimoine "commun ", ce serait donc en faire le lieu même de la régulation des usages, par le dialogue. Pour Dardot et Laval, le commun doit être pensé comme inappropriable en

\footnotetext{
${ }^{1}$ Bortolotto souligne cependant les nouveautés introduites avec la notion de $\mathrm{PCl}$, le troisième critère étant celui de sa recréation permanente : "Cette argumentation théorique en faveur de l'idée de "sauvegarde" fait partie d'un discours patrimonial tout à fait cohérent : si le "folklore" pouvait s'interpréter comme le produit d'une documentation réifiée (un objet d'artisanat conservé dans un musée, une légende transcrite par un ethnologue, un chant enregistré par un ethno-musicologue), le $\mathrm{PCl}$, en revanche, est conçu comme le processus contextuel de recréation de ces éléments par les groupes mêmes qui, à travers ces opérations, activent un processus d'identification culturelle » $\left(2011\right.$, p. 27). Le $4^{\mathrm{e}}$ critère est celui de l'identification et du sentiment de continuité, qui laisse pour sa part peu de place à la dimension inter-culturelle...

${ }^{2}$ Dans le sens de Faro " communauté » est plutôt entendu comme l'ensemble des personnes qui se reconnaissent non pas dans l'objet patrimonial, mais qui se rassemblent dans l'action de patrimonialisation, dans la dynamique démocratique qui consiste à identifier ce à quoi on tient et comment on entend le partager, pour en faire un « commun » (voir plus loin).
} 
ce sens qu'il ne relève ni de la propriété publique ni d'une propriété collective. Il doit être envisagé "comme coactivité, et non comme co-appartenance, copropriété ou copossession » (Dardot et Laval, 2014, p. 48). C'est en ce sens que nous proposons de parler de patrimoine "impropre " (Autant-Dorier et Aubry, 2014) pour saisir la dimension interculturelle dans sa portée politique : " le lieu de manifestation de la différence n'est pas le "propre" d'un groupe ou sa culture. C'est le topo d'un argument. Et le lieu d'exposition de ce topo est un intervalle [...] un être-ensemble comme être-entre : entre les noms, les identités ou les cultures » (Rancière, 1998, p. 122).

Les articles rassemblés ici documentent de façon intéressante la manière dont agissent, en partie ou plus radicalement, ces modalités de dialogue argumenté.

Partant de l'échelle européenne qui leur donne cadre, Biewerth et Brianso s'interrogent sur la façon dont en pratique et localement on élabore du patrimoine en commun. Dans ce contexte, les enjeux de construction du patrimoine par l'Europe sont également des enjeux de construction de l'Europe par le patrimoine ${ }^{3}$. Isabelle Brianso examine comment se traduisent les intentions de la convention de Faro dans la façon dont une communauté locale se relie à ce qu'elle identifie comme du patrimoine. L'enquête montre que les personnes s'attachent au lieu d'abord par les relations qu'ils y ont, les qualités propres du patrimoine n'intervenant qu'en second lieu, et ce sont celles de l'environnement naturel qui sont pointées. Faro apparaît ici comme un dispositif qui permet au Kosovo en construction de prendre place et rang dans l'Europe en rejoignant ses normes, ses conventions et ses formes d'action (projet, observatoire).

L'article de Gesa Biewerth montre comment la notion de " patrimoine commun ", utilisée ici de façon singulière à propos de l'héritage allemand dans les pays de l'Est, constitue davantage un instrument de la construction européenne plutôt qu'une catégorie opératoire. Ce que le terme désigne n'est pas tant défini en regard du passé qu'en regard de ce projet politique (les éléments de patrimoine allemand qui sont sur le territoire de la Russie ne sont pas compris dans ce terme). Les enjeux de l'action définissent les contours pertinents de la notion plus que la réalité historique à laquelle on prétend référer. Les objets et les faits sont, eux, plutôt la trace de la partition de l'ancien empire et des expulsions, ils sont la trace d'un partage, et ils ont donné lieu à des histoires et à des récits nationaux distincts. Suivre les opérations de patrimonialisation, les « retours » en Pologne, le travail de médiation qui s'opère, permet à l'auteure d'étudier comment à distance de cette idée théorique d'un " patrimoine commun " se construisent des espaces de rencontres, de traduction, de réinterprétation. Ce qui se donne à voir est dès lors moins l'objet patrimonial en soi que le travail interculturel qui se réalise.

Partant d'initiatives situées, les articles de Martineau, de Parrino et de Aubry développent à la fois des propositions et une analyse sur la façon dont le patrimoine peut faire lien et favoriser le dialogue interculturel. Qu'il s'agisse de l'expression à travers le conte, du recueil de paroles sur l'hospitalité dans un musée de ville ou d'un projet artistique participatif dans un centre culturel, dans les trois cas, l'attention porte sur la façon dont les contributions de chacun, construites à partir de leurs expériences (notamment migratoires), nourrissent une œuvre commune. L'enjeu n'est pas là pour chacun de défendre ou de faire valoir son propre patrimoine mais de trouver les voies pour accorder ensemble les voix, et faire porter une parole commune.

Barbe et Sévin pointent pour leur part davantage les difficultés à faire du commun à partir d'un fait, devenu objet patrimonialisé : la demande d'abolition de l'esclavage inscrite dans les doléances d'un village au moment de la Révolution française. La logique muséale de la collection et de l'exposition se heurte ici à une triple question : de quoi faut-il se souvenir? Qui se souvient? Et pourquoi? On retrouve là le trouble qu'introduit en d'autres lieux la

\footnotetext{
${ }^{3}$ Guérin (2008, p. 233) note ainsi que "L’Union européenne étant en quête de légitimation, et la construction des identités nationales aux $19^{\mathrm{e}}$ et $20^{\mathrm{e}}$ siècles s'étant fortement appuyée sur des processus de patrimonialisation culturels, on peut émettre I'hypothèse d'un investissement de l'Union dans le patrimoine culturel à des fins de production pragmatique d'un récit culturel européen ". L'analyse des programmes Interreg dans les Alpes conduit à identifier l'émergence de biens communs transfrontaliers qui s'opèrent par le renforcement de l'action publique locale et la construction de codes, normes et valeurs à l'échelle de ces nouveaux territoires. L'Europe contournerait ainsi les identités nationales en produisant non pas une identité européenne mais des identités partagées.
} 
notion de patrimoine immatériel (Bortolotto, 2011) : quelles sont les logiques de sauvegarde qui peuvent valoir ici? Qui est concerné et contribue à instituer du commun et à quelle échelle?

Se pose là la question non seulement des contenus, mais des usages et de la visée des actions patrimoniales, des critères à l'œuvre et de ce que cela fait à ce qu'on prétend «garder sauf » c'est à dire « vivant ».

\section{(Re)considérer les patrimoines, faire entendre des voix profanes et minorées}

Les transformations des conceptions du patrimoine ouvertes par les nouvelles conventions internationales s'inscrivent dans un contexte plus large de transformation des formes de communication et de production culturelle et elles participent à cette transformation. Les États ne jouent plus le rôle central et unificateur qui était le leur, même s'il ne faut pas négliger le poids des grandes institutions culturelles, qui demeure important, en particulier dans le cas français. La possibilité de produire soi-même des images et de les diffuser, comme d'accéder à des ressources et des archives plus facilement, ont encore accentué les flux de circulation culturels et démultiplié leurs usages ${ }^{4}$. Ces nouvelles modalités « d'organisation sociale de la signification » (Hannerz, 2010) se caractérisent en particulier par le développement de démarches hybrides, partagées. Ainsi Tornatore (2004) souligne que, alors que la catégorie de "patrimoine ethnologique " a été définie en France de manière à spécifier la démarche de recherche vis-à-vis des pratiques amateurs, on assiste au contraire avec la reconnaissance du patrimoine immatériel à une reconfiguration des acteurs et des rôles. Les scientifiques " experts " sont considérés au même niveau que les personnes "profanes» mobilisées pour la reconnaissance de leur patrimoine. Cette situation, à mettre en parallèle avec l'émergence concomitante des sciences citoyennes (Roux, Charvolin et Dumain, 2013), fait des "z-acteurs-z-eux-mêmes » les premiers à pouvoir légitimer l'authenticité du patrimoine. Celle-ci n'est plus définie à partir d'une science académique ou par des experts institutionnels, mais en regard de la valeur que lui reconnaissent ceux qui y tiennent et qui ce faisant s'impliquent dans les processus et procédures de sa validation. L'ethno-anthropologue n'est plus celui qui découvre et expose les cultures des autres : il doit composer avec les acteurs eux-mêmes, conscients de leur propre culture, l'explicitant et l'exposant (Fabre, 2001). Morisset et Dieudonné constatent que "d'un régime fondé sur l'authenticité des objets, nous sommes passés à une authenticité des représentations. Ce n'est plus le paysage qui importe, c'est l'usage que chacun en fait. C'est le "paysagement" ou le "dépaysement" » (2006, p. 10). Pour notre part, c'est moins de l'authenticité des représentations que chacun se fait de sa propre culture dont il faudrait parler que de la légitimité des arguments et des usages.

Bortolotto (2011) note à propos de la convention de Faro :

L'importance donnée au rôle des acteurs sociaux dès la phase initiale de définition de cet instrument [...] vient de l'influence de la perspective du public folklore américain [...] ainsi que, sur le plan institutionnel, des tensions qui mettent en question la légitimité des États, et des organisations intergouvernementales, à représenter la société civile [...].

Elle souligne en outre que

Cette approche explicite la portée essentiellement politique des interventions patrimoniales : la production du patrimoine n'est pas conçue simplement comme une production de connaissance mais aussi comme l'expression d'un pouvoir [...]. (2011, p. 32).

Lucia Parrino souligne, dans ce volume, que l'on sort d'un paradigme centré sur la collection et d'un modèle de musée orienté sur son propre fonds pour passer à des musées connectés aux communautés (entendues ici comme populations-publics-territoires). Les institutions se doivent non seulement de fonctionner en écho à leurs publics et de répondre à leurs attentes, mais aussi d'être ancrées, c'est-à-dire d'être pertinentes localement en tant que services communautaires s'occupant des questions d'héritage (" heritage-based community services »).

\footnotetext{
${ }^{4}$ L'espace numérique devient une scène plus ou moins publique de déploiement, permettant à chacun de contribuer à la production mémorielle, voire à la fabrication de véritables espaces patrimoniaux virtuels (Rautenberg et Rojon, 2014).
} 
On retrouve ici les principes fondateurs de "l'éco-musée communautaire " pour lequel le musée est un instrument au service de la communauté pour penser et agir sur les problèmes communs : il sert dans le temps social à remplir une fonction politique (De Varine, 1991). Il n'y a pas là de distinction entre professionnels, chercheurs, publics : chacun est considéré comme acteur et contribue au processus de développement. Car il s'agit ici tout autant de valoriser « le patrimoine » que les personnes qui en sont porteuses. On a affaire ici à des enjeux de considération.

Comme Jean-Louis Tornatore et Sébastien Paul le soulignent concernant les friches industrielles, ces patrimoines souffrent d'ostracisme. Ils sont " l'envers » des formes classiques du patrimoine de la " Haute Culture ». II convient donc d'être attentif aux épreuves qu'ils rencontrent pour comprendre les formes de traitement qu'ils connaissent et nécessitent. "Cette caractéristique est non seulement susceptible d'ouvrir la voie à des cristallisations de la mémoire, mais d'offrir également la possibilité d'élaboration de nouveaux dispositifs de politisation de la mémoire et de la culture qui n'en passent pas par les dispositifs d’État » (Tornatore et Paul, 2003, p. 5). Dans les textes présentés ici, on a affaire à des questions problématiques, à des sujets sensibles : l'esclavage, les recompositions de frontières et les conflits ethniques en Europe, les discriminations... Ces caractéristiques " négatives » altèrent les qualités habituelles recherchées à travers la patrimonialisation : l'identité conçue comme ipséité, la possibilité de valoriser son propre héritage. C'est pourquoi de nouveaux dispositifs doivent être trouvés pour élaborer cet « alterpatrimoine »- caractérisé à la fois par l'altération et l'altérité.

La "Maison de la Négritude et des Droits de l’Homme " (Barbe et Sévin) cristallise des mémoires concurrentes liées à des enjeux politiques qui mettent effectivement à l'épreuve ce dont même il s'agit de faire patrimoine. Ainsi, la valorisation du geste historique du vœu de leurs ancêtres semble conduire les habitants à une autocélébration de leur singularité, qui s'éloigne du souci initial pour leur commune humanité. Ensuite, alors que le musée est identifié et reconnu par des autorités politiques (françaises et africaines), les velléités de demandes de réparation du préjudice subi sont contestées, jugées illégitimes en ce lieu. Enfin, la " négritude » renvoie à une notion aujourd'hui désuète, en référence au passage de Senghor dans le lieu. Elle se donne à voir à travers une collection, dans une exposition à priori classique d'objets. Mais la valeur de ceux-ci ne tient qu'en tant qu'ils sont une trace, un témoignage : celui des cadeaux faits par les visiteurs africains reconnaissants du vœu fait par les habitants et de sa remémoration. Or cette reconnaissance "sanctuarisante " supporte mal une politisation " activatrice » présente dans le même musée : sensibiliser aux formes actuelles de l'esclavage. Les voix sont ici pour le moins discordantes, et la possibilité d’un dialogue interculturel se dérobe.

Dans l'expérience du Centre culturel (CCO) de Villeurbanne dont rend compte Anne Aubry, le travail de création collective agit comme un opérateur qui permet de passer les épreuves et d'accorder les voix pour faire patrimoine commun... du moins le temps d'un spectacle et pour ceux qui le vivent. Mais l'expérience, justement, se reproduit et se ramifie sans cesse au CCO, inventant là une pratique patrimoniale inédite. Alors que l'intention patrimoniale n'est pas première, la description fine du dispositif de création culturelle participatif met en évidence la façon dont s'opère une transmission dans le temps: l'expérience de ce projet, les expériences de vie de chacun, leurs expériences culturelles et artistiques ici et ailleurs...

Dans les ateliers de conte animés par Myriame Martineau, comme pour le projet mené avec le Musée du territoire Vimercatese par Lucia Parrino, l'objectif est également la production par les personnes de leur parole. II s'agit de transformer le rapport entre producteur et récepteur, public et population. Le travail d'écoute et de traduction réalisé s'inscrit dans des dispositifs d'hospitalité singuliers: la trame d'un conte " traditionnel » dans un cas, un méta-design muséographique dans l'autre. Ce que chacun confie est ainsi accueilli par les autres, premier public et producteur d'un travail de mise en récit et de mise en scène collectif qui pourra ensuite être exposé et publicisé, et en premier lieu auprès des proches. Si le conteur n'est plus un passeur d'histoires mais un faiseur de spectacles, comme le signale Martineau, on peut sans doute ajouter que de spectateurs qui connaissaient déjà l'histoire on est passé à un public acteur qui souhaite créer des histoires communes.

Ce souci est au cœur des deux dispositifs étudiés par Isabelle Brianso et Gesa Biewerth. Dans le cas du Kosovo, on se mobilise pour construire un nouveau récit. Et le dispositif de Faro, tel qu'il est mis en place là, vise à permettre la conversion d'une histoire négative des conflits en une dynamique culturelle positive. Le fait que la convention de Faro ne prédéfinisse pas ce qu'est le patrimoine est souligné comme constituant, pour les pays du sud-est de 
l'Europe, une opportunité pour manifester par là leur volonté d'adhérer à l'Europe. En ratifiant la convention, ils ont d'ailleurs contribué à son entrée en vigueur. Dans le cas du " patrimoine commun allemand en Pologne ", les protagonistes œuvrent, du côté de l'Allemagne, pour faire reconnaître ce qu'ils ont subi dans les récits historiques. Du côté de la Pologne, ils rendent lisibles les traces d'une histoire effacée en les inscrivant dans de nouvelles mises en scène et en récit.

Dans les deux cas, mais sans doute est-ce vrai plus largement, c'est le changement de contexte, ou d'éco-système, qui permet ce basculement vers un nouveau régime patrimonial. Si nos rapports au temps et à l'espace ont changé, c'est notre rapport à l'Autre sur lequel il convient d'insister ici. Toutefois, comme nous l'indiquions ci-dessus, il ne s'agit peut-être pas tant de régimes d'authentification (Morisset, 2009) que d'un travail politique de reconsidération. Il s'agit de considérer autrement et nouvellement ce/ceux qui avai(en)t été nié(s) ou négligé(s) et de reconsidérer ce que l'on (en) fait ensemble.

\section{Des chercheurs-praticiens au cœur de la fabrique du patrimoine}

Ce travail de reconsidération ne se fait pas sans bousculer quelques définitions et quelques frontières ${ }^{5}$ : la reconnaissance de la diversité passe ainsi par la diversification des espaces dans lesquels se manifeste une dimension patrimoniale ou dans lesquels prennent sens des références patrimoniales. Les institutions elles-mêmes se trouvent à " sortir des murs " pour mener ce type de démarche et à recourir à des tiers pour les y aider (chercheurs, consultants, partenaires associatifs.... ${ }^{6}$.

Ces nouvelles configurations supposent des négociations et l'établissement d'un dialogue entre espaces institués de production du patrimoine et espaces "communautaires", "collaboratifs", comme le montrent l'article de Lucia Parrino ou les expériences de déplacement du conte dans des lieux d'apprentissage socio-linguistique que développe Myriam Martineau. La dimension patrimoniale est ici une composante parmi d'autres de l'interculturalité.

De ce point de vue, les formes et dispositifs particuliers qui sont activés là relèvent du registre de l'ingénierie interculturelle. Ce qui paraît significatif ici, c'est que ce sont précisément les acteurs qui les ont conçus ou qui y sont impliqués qui en rendent compte et les questionnent. Ainsi, Anne Aubry affirme, après Tornatore, que

Il faut bien que l'anthropologue ou le sociologue quitte le point de vue surplombant ou contraignant de la «machinerie patrimoniale ", descende dans l'arène, s'agrège à la communauté des pratiquants et s'astreigne à l'exigence méthodologique de parcourir avec eux les multiples réseaux de la mémoire et du patrimoine (2006, p. 15).

La chercheure est engagée dans la fabrication de ce qu'elle interroge et qu'elle contribue à nourrir. Son implication dans la recherche-action du CCO sur lui-même contribue à actualiser le rapport du CCO à ce que pourrait constituer son patrimoine : il se constitue en tant qu'objet de recherche partagé... y compris en tant qu'objet polémique. Et c'est bien en suivant les formes rhizomatiques et proliférantes de l'action qu'elle peut en éprouver la consistance et les manifestations sensibles.

Myriame Martineau entrecroise dans son texte deux postures : d'une part, elle analyse les formes d'expressions actuelles du conte au Québec et, d'autre part, en tant que conteuse-collecteuse engagée auprès des populations migrantes, elle renouvelle le répertoire et les usages à partir des paroles de ces dernières. Lucia Parrino propose pour sa part une démarche pour accompagner les musées de ville dans la mise en place de démarches interculturelles. S'appuyant sur le cadre européen des "cités interculturelles", qu'elle module à partir des

\footnotetext{
${ }^{5}$ Par exemple, Bortolotto souligne que « dans d'autres pays, comme la France, la notion de communauté, vue comme facteur de désagrégation de la cohésion sociale [...] met dans l'embarras les fonctionnaires de l'État chargés de la mise en œuvre de la Convention. » (2011, p. 30).

${ }^{6}$ Le projet même du GIS IPAPIC illustre bien ce mouvement en même temps que cette nécessité (Hatzfeld, 2015). À titre personnel, c'est bien cette invitation à faire se rencontrer des acteurs multiples qui m'a permis de " découvrir » le patrimoine à partir de préoccupations plutôt orientées sur les dispositifs de l'intervention sociale et des questionnements portant sur la reconnaissance des minorités et l'action communautaire.
} 
pratiques observées dans les petits musées, elle propose une adaptation pragmatique. Enfin, elle exerce un retour réflexif sur la mise à l'épreuve concrète de ce "méta-désign » au MUST (Museo del Territorio Vimercatese). Noël Barbe et Jean-Christophe Sévin montrent comment, mandatés pour accompagner la réflexion sur le projet muséal, ils se retrouvent pris dans un objet hybride qui ne peut être redéfini. Quant à Isabelle Brianso, elle combine une analyse globale des principes de Faro avec son rôle d' « experte» recrutée pour alimenter l'observatoire qui constitue l'instrument principal de l'effectuation de cette convention. Seule Gesa Biewerth semble se situer dans une posture de recherche plus distanciée vis-à-vis de son objet.

Les pratiques patrimoniales, auxquelles nous associons les travaux qui s'y rapportent, apparaissent ainsi comme des "objets chevelus" (Latour, 1995), en ce sens qu'ils supportent mal les partages que l'on a tenté de leur appliquer entre science et croyance, rationalité et émotion. Le programme du Lahic sur les émotions patrimoniales, conduit depuis 2001, en décline les multiples ramifications (Hottin, 2011). Si elles sont propices à la controverse, elles sont également des espaces d'expérimentation.

Nous voudrions souligner que ces points de vue conduisent à décrire les formes de réciprocité qui s'établissent et les espaces de convivialité qui se créent. Martineau parle de "lien de mémoire ". Cette attention portée à la qualité de la relation n'est pas anecdotique: ces expériences touchent à des questions sensibles, là où des problèmes se posent pour les gens. On s'inscrit ici dans le registre de la civilité des relations, des possibilités de cohabitation dans le monde commun au sens le plus concret du terme. Il n’y a pas nécessairement ici « montée en politique » du côté de la citoyenneté abstraite, mais on se saisit des questions ordinaires qui se posent dans le vivre ensemble. On tente, de ce point de vue, d'explorer des voies de cohabitation.

\section{Ce que l'interculturalité fait faire au patrimoine}

Nous voudrions examiner pour terminer ce à quoi ouvrent ces explorations. Le renouveau de la muséographie d'une part et, d'autre part, la multiplication des projets sociaux, culturels et scientifiques qui travaillent la mémoire, les traces et la friche ont considérablement transformé les modalités d'exposition et de rapport aux " objets » patrimoniaux. La notion même de patrimoine immatériel, nous l'avons dit, impose de concevoir autrement cette notion. Parallèlement, on observe un renouveau des modalités de recherche. Le GIS IPAPIC a fonctionné à ce titre comme un laboratoire pour œuvrer à ce renouvellement. Les contributions présentées ici contribuent à l'identification de quelques caractéristiques du patrimoine à l'épreuve de l'interculturalité.

Les actions conduites non seulement visent à faire patrimoine, mais cherchent à " faire faire " à travers lui : faire l'expérience de la rencontre, mobiliser les contributions de chacun, créer de nouvelles significations culturelles, faire participer à la démocratie...

Afin d'approcher plus concrètement la dimension participative de l'activité humaine, il convient de passer par la notion d'expérience. [...] II faut comprendre l'expérience comme un processus conscient d'adaptation à une situation troublée, problématique, bloquée. II s'agit, explique Dewey, non du fait de s'agiter, ni à l'inverse du simple fait d'être affecté par une excitation extérieure, mais de découvrir des moyens disponibles dans telle situation problématique afin de supprimer le handicap à l'action que provoque tel ou tel événement extérieur. Dans les situations d'équilibre, les moyens et les fins sont continus les uns aux autres. Il y a trouble au contraire à partir du moment où, soit les moyens ne sont pas disponibles pour atteindre les fins que vise l'individu, soit l'individu n'est plus dans une situation telle qu'il puisse articuler des fins capables de canaliser son action, soit tous les moyens qu'il utilise s'avèrent non pertinents, soit la situation lui est devenue si étrangère qu'il n'y discerne plus ni moyens ni fins. (Zask, 2013)

Nous avons relevé plus haut que nous nous trouvions dans une situation de basculement de régime liée aux transformations du rapport à l'altérité. Confrontés au déséquilibre, il s'agirait de s'engager dans de nouvelles expériences, de participer à construire un nouvel équilibre. La tâche n'est cependant pas aisée... 
L'idée d'activer la cité interculturelle a été empruntée par Lucia Parrino à l'occasion de la conférence internationale de l'association des musées de ville (2006) qui avait pour titre " activer la ville " ${ }^{7}$. L'objectif est d'avoir un rôle dans l'amélioration de la qualité de vie. Pour cette chercheure, l'activation de la dimension « interculturelle " qui serait développée à travers le musée pourrait fonctionner dans la ville même s'il n'y a pas de projet politique « d'en haut " qui vise cette reconnaissance. Dans le projet MUST, c'est grâce à l'orientation même du musée et à la volonté forte du conseil local et au rôle joué par l'association de migrants que cela fonctionne. Elle constate cependant que cette activation reste éphémère: la collection elle-même ne change pas, la formation et la sensibilisation des acteurs du musée à l'interculturalité " ne sont pas la priorité » et l'action culturelle n'est pas devenue un axe transversal. Si ces limites sont d'abord imputées aux manques de moyens, l'auteur identifie plus fondamentalement une critique que l'on peut adresser aux politiques de développement de l'interculturel comme à celles qui valorisent la participation des habitants: les institutions ont tendance à se contenter d'actions ponctuelles qui produisent un "empowerment" factice. Ces sollicitations sans suite comportent ainsi des ambivalences et provoquent des tensions sociales, elles peuvent même avoir des effets paradoxaux de " disempowerment » et de " gentrification ».

Les projets conduits au CCO relèvent eux aussi du registre de l'activation citoyenne et se donnent pour visée de permettre les rencontres interculturelles. L'hypothèse de Anne Aubry est que la création artistique interculturelle permet de faire patrimoine autrement, selon un registre prospectif et une logique de recréation constante. L'originalité de l'article tient à la conception du patrimoine qui est proposée, en référence à des approches du concept qui ont davantage été développées du côté du patrimoine naturel ${ }^{8}$. L'enjeu est ici de comprendre " le travail du vivant en coopération (Negri) ». II s'agit d'un " rassemblement qui plutôt que de faire identité est mouvement ", supposant des activations et réactivations permanentes. Les référents culturels mobilisés sont entendus comme étant une forme d'expression des personnes plus que des objets ou des contenus prédéfinis, déjà disponibles. De même, ce qui «fait tradition » tient davantage dans les manières de faire ensemble ou même de chercher à faire ensemble plutôt que dans un modus operandi qu'il suffirait d'appliquer. D'ailleurs, si les acteurs se réfèrent à la convention de Faro ou à la déclaration sur les droits culturels de Fribourg, c'est davantage comme matière à réflexion que comme guide de bonnes pratiques.

La question posée par le texte de Isabelle Brianso est pour sa part de voir si et comment la convention de Faro permet de faire vivre quelque chose à Junik. Dans quelle mesure peut-on activer « du haut » des dynamiques de participation locale?

Comme le note Macchiarella à propos du Canto a tenore, " c'est seulement en connaissant parfaitement un mécanisme de créativité musicale (et plus généralement une expression immatérielle) dans sa transformation continue qu'on le sauvegarde réellement. " (cité dans Bortolotto 2011, p. 28). Pour lui, ne pas reconnaître la performance, c'est dénaturer une pratique où la relation compte plus que son objectivation. Le propos de Myriame Martineau s'inscrit dans cette perspective: la question qu'elle pose est celle de la pertinence de la voix des conteurs et conteuses contemporains. L'expression importe plus que le corpus. La transmission se fait non seulement par les contenus de discours, mais aussi par ce qu'on va vivre dans ce temps de partage. Le conte est compris ici comme un art de l'expérience. Or, selon Dewey "L'activité artistique serait l'un des moyens par lesquels nous entrons, par l'imagination et les émotions [...], dans d'autres formes de relations et de participations que les

\footnotetext{
${ }^{7}$ La notion d'activation renvoie aux tendances que l'on peut observer plus largement dans le champ des politiques publiques et notamment des politiques sociales et urbaines, de l'activation des dépenses passives qui lie les mécanismes de solidarité à des contreparties telle que l'inscription dans des projets d'insertion de la part des « bénéficiaires » (Revenu de solidarité active) aux formes d'activation citoyenne prônées par l'entremise des divers dispositifs participatifs à l'échelle européenne comme à celle du quartier. L'activation des personnes - publics "éloignés des institutions ", notamment immigrés - comporte une ambivalence certaine. Pour le meilleur il s'agit de retrouver l'estime de soi et de gagner en pouvoir d'agir. Pour le pire on tombe dans l'injonction au projet et la responsabilisation individualisante.

${ }^{8}$ C'est à une conception du patrimoine comme "bien commun " que cette approche renvoie. Micoud (2000) a montré que l'usage de ce terme permet d'insister sur la dimension publique de la définition du patrimoine. Pour Sgar (2010), l'enjeu est de débattre pour définir le Bien au sens éthique et politique, et cela engage la responsabilité collective. Elle note que « la notion de bien commun trouve sa place dans des démarches, en particulier les démarches participatives, mettant l'accent sur la projection dans l'avenir, à travers des scénarii, des représentations collectives du paysage de demain [...] ».
} 
nôtres» (2010, p. 382). Que ce soit la capacité de se mettre à la place de l'autre, d'envisager des modes de vie alternatifs ou d'entrer en relation originale avec certaines parties de la nature ou de notre environnement matériel, les œuvres d'art peuvent être des points de convergence entre nous et le monde. En ce sens, "l'imagination est le principal instrument du bien » (2010, p. 397). L'expérience esthétique permettrait ainsi d'entrer en politique en ouvrant des perspectives communes de formes de vie meilleures. L'expérience patrimoniale pourrait également y contribuer dans la mesure où elle ré-active bien une expérience esthétique.

Gesa Biewerth met également en évidence l'importance de ce qui est activé (là où Anne Aubry parlerait d'« êtredevenir ») :

Le véritable rapprochement ne se fait pas par le patrimoine proprement dit, mais au deuxième degré par la réalisation de projets permettant aux Allemands et aux Polonais de renouer ensemble et d'une manière nouvelle avec l'ancien en trouvant des compromis, en attribuant un sens nouveau et partagé au patrimoine, voire en le faisant naître ou renaître.

L'intérêt de cette enquête est qu'elle permet de montrer que les personnes qui s'attachent à ce patrimoine allemand en Pologne ne sont pas comme on le pense des revanchistes, passéistes, mais qu'elles réalisent très concrètement dans leurs pratiques des rencontres interculturelles, jouant le rôle de médiateurs culturels. L'auteure conclut sur le fait que l'argument du « patrimoine commun » est un argument « faible ».

Si, du point de vue de l'analyse critique, on ne peut qu'approuver cette lecture, nous voudrions insister sur ce qui est dit là. Notre hypothèse est que cette faiblesse serait justement ce qui peut permettre de déployer l'action et de faire patrimoine interculturel ${ }^{9}$. De même qu'au CCO on peut observer qu'il ne s'agit pas d'un patrimoine fort, d'emblée défini et approprié, ici cette faiblesse ouvre vers le présent et l'avenir plutôt que d'être une réification du passé. De fait, le " patrimoine commun " n'est pas ici un concept, mais bien un postulat discutable et discuté, c'està-dire une hypothèse qui permet d'agir et de faire lien.

C'est à cette condition, nous semble-t-il, que l'on peut sortir de processus d'identification et de réification des identités pour s'ouvrir à l'altérité. La forme "musée ", pour sa part, risque toujours d'échouer à cela dès lors qu'elle clôt l'expérience. Jean-Christophe Sevin et Noël Barbe montrent bien comment dans le cas du musée de l'esclavage on est à la frontière, et dans une perpétuelle indécision, entre identification et désidentification. Le texte de doléances invite à la subjectivation politique au sens de Rancière, puisqu'il y a bien " une démonstration : qui suppose un autre auquel on s'adresse, même si cet autre refuse la conséquence. Elle est la constitution d'un lieu commun. Elle ouvre la polémique, le traitement du tort, et la démonstration de l'égalité » (1998, p. 118). Mais I'on reste dans la monstration qui ne conduit pas à faire participer et à partager des expériences. Les " autres » ne sont que des visiteurs dûment catalogués, invités à voir ce qui est exposé et qui ne peuvent exprimer là que leurs remerciements, jamais leur parole. Fait révélateur : ce que l'on conserve d'eux ce sont des objets... sans valeur!

\section{À l'inverse :}

La performativité qui est dans ce contexte l'action qui fait vivre le sens et la parole, la mise en acte et en corps de la narrativité, est une revanche de la négation de l'existence ou de l'effacement qui fut le lot du minorisé. L'expérience du dehors du monde, qui est celle de l'exclusion, passe par l'expérience performative d'une entrée ritualisée dans le monde, laquelle permet, sur le plan symbolique, la sortie du miroir, du faux miroir de l'altérisation. (Saillant, 2014)

À l'aune de ces observations, les défis à relever, pour être précisés, n'en sont pas pour autant relevés. Le patrimoine s'occupait du passé, voilà que nous le découvrons prospectif et performatif, espace d'invention de la culture. II caractérisait un groupe dans le temps, nous montrons que son opérativité tient à sa faiblesse et au fait

\footnotetext{
${ }^{9}$ Michel Rautenberg, dans la recherche que nous avons conduite ensemble au CCO (Autant-Dorier et Aubry, 2014), avait comparé l'expérience de Villeneuve d'Acsq et celle du CCO en parlant de patrimoine faible: "Ce patrimoine, on peut le caractériser de "faible" car, en référence aux liens faibles de Granovetter, il apparaît d'autant plus puissant qu'il est peu institué, qu'il se construit à bas bruit, ce qui permet de multiplier les effets de levier, les liaisons avec d'autres collectifs sans empiéter sur leurs singularités... II est efficace car ouvert, n'enferme pas sur des récits canoniques ou des images iconiques ou des lieux à protéger. "
} 
qu'il ne peut être approprié. II était authentifié, classé, protégé, voilà qu'il devient revendiqué, participatif, pratiqué.

\section{Bibliographie}

Autant-Dorier, C. et Aubry, A. (2014). Pratiques (inter)culturelles et institution d'un patrimoine. 50 ans d'activité au CCO-Jean Pierre Lachaize (Villeurbanne) (Rapport pour le ministère de la Culture et de la Communication). https://halshs.archives-ouvertes.fr/halshs-00960079

Bhabha Homi, K. (2007). Les Lieux de la Culture - Une théorie postcoloniale (trad. Françoise Bouillot). Paris : Payot.

Bortolotto, C. (2011). Le patrimoine culturel immatériel. Enjeux d'une nouvelle catégorie. Paris : Éditions de la Maison des Sciences de l'Homme.

Cohen-Emerique, M. et Rothberg, A. (2015). La méthode des chocs interculturels. Rennes : Presses de l'EHESP.

Dardot, P. et Laval C. (2014). Commun. Essai sur la révolution au XXI siècle. Paris : La Découverte.

Davallon, J. (2006). Le Don du patrimoine : Une approche communicationnelle de la patrimonialisation. Paris : Hermès-Lavoisier.

Davallon, J. (2013). Du patrimoine à la patrimonialisation. http://crdp.ac-paris.fr/preacpatrimoinesetdiversite.

De Varine, H. (1991). L'initiative communautaire, recherche et expérimentation. Macon : W/MNES.

Dewey J. (2010). L'art comme expérience (trad. J. P. Cometti et collab.). Paris : Gallimard. (1 ${ }^{\mathrm{e}}$ éd. 2005; ouvrage original publié en 1934 sous le titre Art as experience)

Fabre, D. (2001). L'histoire a changé de lieux. Dans D. Fabre et A. Bensa (dir.), Une histoire à soi. Figurations du passé et localité. Paris : Maison des sciences de l'Homme.

Goguikian Ratcliff, B. et Rossi, I. (2014). Santé mentale et sociétés plurielles, Alterstice, 4(2), 3-12.

Guérin, M.-A. (2008). Le patrimoine culturel, instrument de la stratégie de légitimation de l’Union européenne. L'exemple des programmes Interreg. Politique européenne, 2(25), 231-251.

Hannerz, U. (2010). La complexité culturelle. Études de l'organisation sociale de la signification (trad. Alain Battegay, Pascale Joseph, Daniel Mandagot et Hervé Maury). Édition À la croisée. (ouvrage original publié en 1992 sous le titre Cultural complexity)

Hatzfeld, H. (2015). Regards décalés sur des patrimoines silencieux. Paris : Ateliers Henry Dougier.

Hobsbawm, E. et Ranger, T. (1983). The Invention of Tradition. Cambridge : Cambridge University Press.

Hottin, C. (2011). Émotions patrimoniales : retour sur les détours d'un programme de recherches. Livraisons de I'histoire de l'architecture, 22, 59-86.

Latour, B. (1995). Notes sur certains objets chevelus. Nouvelle revue d'ethnopsychiatrie, 27, 21-36.

Micoud, A. (1995). Le Bien Commun des patrimoines. Dans Patrimoine culturel, patrimoine naturel, actes du colloque de l'École Nationale du Patrimoine, Paris, 12 et 13 décembre 1994. Paris : La Documentation française.

Micoud, A. (1999). Patrimoine et légitimité des territoires. De la construction d'un autre espace et d'un autre temps commun. Dans F. Gerbaux (dir.), Utopie pour le territoire : cohérence ou complexité? (p. 53-65). Paris : Éditions de l'Aube.

Micoud, A. (2000). Patrimonialiser le vivant. Espaces Temps, 74-75, 66-77. (Transmettre aujourd'hui. Retour vers le futur)

Morisset, L. et Dieudonné, P. (dir.). (2006). Patrimoines pour le XXI siècle. Regards du Québec et de la Bretagne. Québec : Nota bene.

Alterstice - Revue Internationale de la Recherche Interculturelle, vol. $5, n^{\circ} 2$ 
Morisset, L. (2009). Des régimes d'authenticité. Essai sur la mémoire patrimoniale. Rennes : Presses universitaires de Rennes.

Rancière, R. (1998). Aux bords du politique. Paris : Folio.

Rautenberg, M. (2003). La rupture patrimoniale. Édition À la croisée.

Rautenberg, M. (2006). La valorisation culturelle des mémoires de l'immigration, l'exemple de la région RhôneAlpes. Écarts d'identité, 108.

Rautenberg, M. et Rojon, S. (2014). Hedonistic Heritage: Digital Culture and Living Environment. Cultura: International Journal of Philosophy of Culture and Axiology, 11(2), 59-81.

Roux, J., Charvolin, F. et Dumain, A. (dir.) (2013). Passions cognitives. Paris : Éditions des Archives Contemporaines.

Saillant, F. (2014). Anthropologie et performativité : transformations et connexions [en ligne]. Cultures-Kairós, Les numéros. http://revues.mshparisnord.org/cultureskairos/index.php?id=905.

Sgard, A. (2010). Le paysage dans l'action publique : du patrimoine au bien commun [en ligne]. Développement durable et territoires, 1(2). http://developpementdurable.revues.org/8565

Tornatore, J.-L. (2004). La difficile politisation du patrimoine ethnologique. Terrain, 42, 149-160.

Tornatore, J.-L. (2006). Les formes d'engagement dans l'activité patrimoniale. De quelques manières de s'accommoder au passé. Dans V. Meyer V. et J. Walter (dir.), Formes de l'engagement et espace public. Nancy : Presses Universitaires de Nancy. https://halshs.archives-ouvertes.fr/halshs-00122998

Tornatore, J.-L. et Paul, S. (2003). Publics ou populations? La démocratie culturelle en question de l'utopie écomuséale aux "espaces intermédiaires". Dans O. Donnat et P. Tolila (dir.), Le(s) public(s) de la culture. Politiques publiques et équipements culturels (p. 299-307). Paris : Presses de sciences po.

Unesco (2003). Convention pour la sauvegarde du patrimoine culturel immatériel 2003 [en ligne]. http://portal.unesco.org/fr/ev.php-URL_ID=17716\&URL_DO=DO_TOPIC\&URL_SECTION=201.html

Zask, J. (2011). Participer. Essai sur les formes démocratiques de la participation. Paris : Le Bord de l'eau.

Zask, J. (2013). Pragmatisme et participation. Dans I. Casillo, R. Barbier, L. Blondiaux, F. Chateauraynaud, J. M. Fourniau, R. Lefebvre, C. Neveu et D. Salles (dir.), Dictionnaire critique et interdisciplinaire de la participation. Paris : GIS Démocratie et participation. http://www.dicopart.fr/fr/dico/pragmatisme-etparticipation. 